\title{
Candida albicans ventriculoperitoneal shunt infections: Immunocompetent preterm pediatric case series and an extensive review of literature
}

\author{
Atta U Bhatti ${ }^{1}$, Maria A Parekh ${ }^{1 *}$, Mahin Amanullah ${ }^{2}$ and Bernd Daeubler ${ }^{3}$ \\ ${ }^{1}$ Department of Neurosurgery, South City Hospital, Pakistan \\ ${ }^{2}$ Bahria University Medical \& Dental College, Pakistan \\ ${ }^{3}$ Radiologie Nordost, St. Gallen, Switzerland
}

\begin{abstract}
Cerebrospinal fluid shunt devices are increasingly being used for decompression, and despite excellent results, obstruction and infection are concerning complications, with underlying fungal etiology a rare consideration. In this study, we focus on pediatric shunt infections caused by Candida albicans in an extensive literature review and by reporting two additional cases. Very limited information regarding diagnosis and treatment of the Candidal shunt infections is available. Prematurity and use of broad spectrum antibiotics have been postulated as risk factors, and a CSF analysis prior to surgical placement of the shunt should be implemented as standard practice. Based on our cases, we suggest establishing the diagnosis based on a single positive fungal culture, and to initiate with intravenous and intraventricular antifungal therapy for two weeks, switching to oral antifungal medication for a total of 43 days in an outpatient setup without immediate ventriculoperitoneal shunt removal.
\end{abstract}

Abbreviations: CSF: Cerebrospinal Fluid, EVD: External Ventricular Drain, ICP: Intracerebral Pressure, LP: Lumbar Puncture, MRI: Magnetic Resonance Imaging, VP: Ventriculo-Peritoneal

\section{Introduction}

Cerebrospinal fluid shunt devices are commonly indicated for the treatment of hydrocephalus in infants, and have served to improve survival and reduce mortality. However, ventriculoperitoneal shunt infection is a serious complication, commonly with an underlying bacterial etiology [1,2]. Candidal infections remain a rare complication of neurosurgery [3]. but are associated with a significant mortality, morbidity, and cost. The emerging importance of Candida as a nosocomial pathogen prompted us to undertake a review of published literature over the last sixty years, revealing a total of 23 cases of Candida albicans shunt infections in children under the age of two [4-13]. We report an additional two cases of Candida albicans ventriculoperitoneal shunt infection in immunocompetent preterm infants, and discuss the unique features and treatment of our cases in light of those reported previously.

\section{Cases}

\section{Case 1}

A four-month old female infant presented to the Emergency with complaints of fever, cough, squint and increasing head circumference one month after the insertion of a ventriculoperitoneal shunt. Her past medical history was significant for preterm delivery and neonatal sepsis with no seizures. At the age of three months, an MRI was performed for increasing head circumference and vomiting, establishing a diagnosis of non-communicating hydrocephalus, for which a ventriculoperitoneal shunt was placed. This procedure was performed elsewhere, and a CSF analysis was not performed prior to surgery. On presentation at our hospital a month later, her initial CSF analysis showed occasional leukocytes, a protein concentration of 290 $\mathrm{mg} / \mathrm{dl}$, a glucose concentration of $22 \mathrm{mg} / \mathrm{dl}$, and yielded C. albicans sensitive to amphotericin-B, Fluconazole and Voriconazole. The culture was positive only this once after which serial CSF examinations revealed sterility with persistence of high protein and low glucose levels. Treatment with antibiotics, intrathecal amphotericin- $B$ and Fluconazole was promptly started, and her shunt was removed 2 weeks after initial presentation. Intermittent serial fontanelle taps were performed alongside, in progressively increasing intervals, to address high pressure-related symptoms. After 43 days of antifungal therapy, she recovered completely without necessitating reinsertion of a shunt device, and has had no signs of elevated intracerebral pressure to date.

\section{Case 2}

Around the same time, a five-month old male infant presented to our clinic with inflammation and purulent discharge along the tract of a previously inserted ventriculoperitoneal shunt. Past medical history was significant for preterm delivery and neonatal sepsis. He had been subsequently diagnosed with hydrocephalus for which a ventriculoperitoneal shunt was placed at his local hospital without

Correspondence to: Parekh MA, Department of Neurosurgery, South City Hospital, Street 1, Block 3, Shahrah-e-Firdousi, Karachi 75600, Pakistan, Tel: (92)21-35862301-3, Fax: (92)21-35878639, E-mail: m.afridi.parekh@gmail.com

Key words: candida albicans, ventriculoperitoneal shunt, infection

Received: January 14, 2017; Accepted: February 07, 2017; Published: February 10,2017 
prior CSF analysis. Since his presentation at our clinic appeared predominantly bacterial, the VP shunt was immediately removed and multiple antibiotics were started. However, C. albicans was isolated from the first culture of CSF, with a leukocyte count of $200 / \mathrm{cm}^{3}$, a protein concentration of $356.5 \mathrm{mg} / \mathrm{dl}$, and a glucose concentration of 18 $\mathrm{mg} / \mathrm{dl}$. Intrathecal amphotericin-B was administered for 43 days along with Vancomycin, and the ventriculoperitoneal shunt was eventually re-inserted due to persistently elevated intracerebral pressure (Table 1).

\section{Discussion}

A thorough review of published English literature over the last sixty years from 1956 to 2014 revealed a total of 53 cases of Candidal ventriculoperitoneal shunt infections, suggesting that Candida albicans remains a rare causative organism complicating neurosurgical shunt procedures [3]. Of the 39 cases described, 23 were children under the age of two years [4-13]. Females accounted for $68.8 \%(11 / 16)$, 13 were preterm infants, and a history of prior use of antibiotics was seen in six of nineteen reported cases. The most common presenting complaints were fever ( 8 of 20 infants), vomiting (6 of 20 infants), and hydrocephalus ( 4 in 20 infants). Outcomes were not reported for three of the 23 children; the rest were treated successfully. Therapy included shunt removal and administration of systemic antifungals for most of the patients. Amphotericin-B was the most commonly prescribed antifungal (75\%), with five patients receiving it via both the intravenous and intraventricular route. Baradkar [4] treated his four cases of $C$. albicans shunt infection with systemic amphotericin without clarifying if the shunt was removed. Other drugs that were used successfully were Fluconazole [7], Flucytosine [12], gentian violet [5] and Caspofungin [10] (Table 2).

Although Candida constitutes the normal flora of the genitourinary tract and skin [14], certain predisposing factors have been implicated in its pathological acquisition. These include indwelling catheters, steroids, immunosuppression, malignancy, cerebrospinal fluid leakage and bowel perforation or abdominal surgery $[1,3,4,11,14-16]$. Prematurity and the use of prior antibiotics have also been identified as risk factors, and are congruent with our observations.

Our first case presented with fever and hydrocephalus while the other had a predominantly bacterial presentation, with mucopurulent discharge along the tract of the ventriculoperitoneal shunt. CSF was promptly sent for cytology and culture, and antibiotics were started. In both cases, only the first CSF culture was positive for Candida albicans with increased protein and decreased glucose levels; serial CSF examinations revealed sterility. Initial literature review revealed insufficient data, in terms of well-defined guidelines and lack of a proper criterion, to diagnose a case of Candidal shunt infection, and its optimal treatment. We suggest that a high index of suspicion should be maintained, that diagnosis should be made on a single positive culture, and treatment should be promptly initiated if the patient is symptomatic with an indwelling device and deranged CSF parameters. This is comparable to recommendations made by Geer et al. [17].

Generally, treatment includes shunt removal, administration of antifungal therapy and placement of a new shunt $[2,6,16]$. However, our cases were managed differently. Case 1 was started on intraventricular and intrathecal amphotericin-B with the shunt in situ initially for two weeks. Instead, serial fontanelle taps for raised intracerebral pressure (ICP) were performed, with intervals lengthening over time, i.e. on days 3,7 , and 18 . Eventually, all high pressure signs settled and the infant had a shunt-less outcome. The CSF became sterile within the first week but medical therapy was continued for a total of 43 days, due to persistence of low CSF glucose and high protein levels. Based on this observation, we propose that the indication for shunt reinsertion should not be solely based on CSF protein levels, but should also assess and incorporate the patient's clinical response. Serial anterior fontanelle taps proved to be a cost-effective intervention in our limited resources setting; also preventing serious complications such as external ventricular drain (EVD) related overdrainage, and reinfection. A fontanelle tap works by improving CSF flow dynamics by exerting an intermittent flow challenge and thus, may result in an ideal shunt-less resolution of hydrocephalus, as in our case.

On the other hand, the seemingly bacterial presentation of Case 2 necessitated immediate shunt removal and administration of antibiotics, including intrathecal Vancomycin, Amikacin and systemic antibiotics. A positive Candidal culture of the cerebrospinal fluid was unexpected, for which we recommended administration of amphotericin and Fluconazole. After several negative bacterial culture reports, ventriculoperitoneal shunt revision was performed to treat persistent hydrocephalus, with outpatient serial fontanelle taps to address raised ICP signs. The persisting high protein levels despite negative cultures were thought to be a reaction to the intrathecal antibiotics. Fever and lethargy were dealt successfully with Fluconazole administered for 43 days. Our study shows that shunts exposed to fungal infections behave similarly to patients with Mycobacterium tuberculosis infection, where the shunts are left in situ during treatment. Based on this, we suggest that shunt removal is not mandatory in fungal infections, as opposed to some studies $[6,15,18]$ that state otherwise, and that these infections can be treated in an outpatient setup. Although administration of intrathecal antifungals is deemed controversial [17], the two new cases reported in our study have had rapid and absolute recovery with it.

Table 1. Summary of clinical characteristics of our 2 cases of Candidal VP shunt infection.

\begin{tabular}{|l|c|c|}
\hline \multicolumn{1}{|c|}{ Case 1 } & Case 2 \\
\hline Age & 5 months & Male \\
\hline Gender & Female & Yes \\
\hline Preterm & Yes & Yes \\
\hline Sepsis & Yes & Yes \\
\hline History of broad spectrum antibiotics use & Yes & Yes \\
\hline Hydrocephalus & Yes & Yes \\
\hline VP shunt placement & Not done & Not done \\
\hline CSF analysis prior placement & Fever, hydrocephalus, low birth weight \\
\hline Presenting complaints & C. albicans (only 1st culture) & C. albicans (only st $^{\text {st }}$ culture) \\
\hline CSF culture & No & Yes \\
\hline Immediate shunt removal & Antifungal therapy & Antibiotics followed by antifungal \\
\hline Treatment & No & Yes \\
\hline Shunt Replacement & & \\
\hline
\end{tabular}


Table 2. All cases of Candida albicans shunt infection reported to date in children $\leq 2$ years

\begin{tabular}{|c|c|c|c|c|c|c|c|c|}
\hline Pt. No. & Reference & Age/ Sex & Underlying condition & Clinical presentation & $\begin{array}{l}\text { Prior bacterial } \\
\text { infection }\end{array}$ & CSF culture & $\mathbf{R x}$ & Outcome \\
\hline 1 & [9] & Child & Shunt revision & NR & NR & C. albicans & NR & NR \\
\hline 2 & {$[5]$} & $2 \mathrm{mo} / \mathrm{F}$ & Lumbosacral meningomyelocele & Hydrocephalus & + & C.albicans & Ventricular aspiration + shunt removal & Cured \\
\hline 3 & {$[5]$} & $2 / \mathrm{F}$ & Communicating hydrocephalus & Meningoencephalitis & + & C. albicans & Gentian violet + shunt removal & Cured \\
\hline 4 & [12] & $2 \mathrm{mo} / \mathrm{M}$ & IVH & Hydrocephalus & NR & C. albicans & $5 \mathrm{FC}+$ ivt antibiotic + shunt removal & Cured \\
\hline 5 & {$[8]$} & NR & Perforated bowel wall & NR & NR & C. albicans & NR & NR \\
\hline 6 & {$[8]$} & NR & Spontaneous shunt infection & NR & NR & C. albicans & NR & NR \\
\hline 7 & {$[15]$} & $47 \mathrm{~d} / \mathrm{F}$ & Prematurity, aqueductal stenosis & Hydrocephalus & + & C. albicans & amB iv + shunt removal & Cured \\
\hline 8 & {$[15]$} & $19 \mathrm{mo} / \mathrm{F}$ & IVH & Meningoencephalitis & + & C. albicans & amB iv + shunt removal & Cured \\
\hline 9 & {$[15]$} & $3 \mathrm{mon} / \mathrm{F}$ & Congenital hydrocephalus & Hydrocephalus & + & C. albicans & amB iv + amB ivt + shunt removal & Cured \\
\hline 10 & [15] & $1 \mathrm{mo} / \mathrm{F}$ & Meningomyelocele & Hydrocephalus & + & C. albicans & amB iv + amB ivt + shunt removal & Cured \\
\hline 11 & {$[7]$} & $2 \mathrm{mo} / \mathrm{F}$ & IVH & Fever, shunt malfunction & - & C. albicans & Fluconazole iv + shunt removal & Cured \\
\hline 12 & {$[6]$} & $6 \mathrm{mo} / \mathrm{F}$ & Prematurity, hydrocephalus & Poor activity level & - & C. albicans & amB iv + shunt removal & Cured \\
\hline 13 & {$[6]$} & $5 \mathrm{mo} / \mathrm{M}$ & Prematurity, hydrocephalus & Poor activity level & - & C. albicans & amB iv + ivt + shunt removal & Cured \\
\hline 14 & {$[6]$} & $6 \mathrm{mo} / \mathrm{M}$ & Prematurity, hydrocephalus & Shunt malfunction & - & C. albicans & amB iv + shunt removal & Cured \\
\hline 15 & {$[6]$} & $3 \mathrm{mo} / \mathrm{M}$ & Prematurity, hydrocephalus & Fever, shunt malfunction & - & C. albicans & amB iv + shunt removal & Cured \\
\hline 16 & [6] & $8 \mathrm{mo} / \mathrm{M}$ & Prematurity, hydrocephalus & Fever, shunt malfunction & - & C. albicans & amB iv + shunt removal & Cured \\
\hline 17 & [11] & $7 \mathrm{mo} / \mathrm{F}$ & IVH & Abdominal symptoms & - & C. albicans & $\mathrm{amB}$ iv $+\mathrm{amB}$ ivt + shunt removal & Cured \\
\hline 18 & [11] & $2 / \mathrm{F}$ & Choroid plexus papilloma & Abdominal symptoms & - & C. albicans & amB iv + amB ivt + shunt removal & Cured \\
\hline 19 & [4] & NR & Prematurity, hydrocephalus & Fever, vomiting & - & C. albicans & AmB iv & Cured \\
\hline 20 & {$[4]$} & NR & Prematurity, hydrocephalus & Fever, vomiting & - & C. albicans & $\mathrm{AmB}$ iv & Cured \\
\hline 21 & [4] & NR & Prematurity, hydrocephalus & Fever, vomiting & - & C. albicans & AmB iv & Cured \\
\hline 22 & [4] & NR & Prematurity, hydrocephalus & Fever, vomiting & - & C. albicans & AmB iv & Cured \\
\hline 23 & {$[10]$} & $8 \mathrm{mo} / \mathrm{M}$ & Prematurity, IVH grade III & Hydrocephalus & - & C. albicans & removal of Omaya reservoir + caspofungin & Cured \\
\hline
\end{tabular}

\section{Conclusions}

In conclusion, the authors recommend maintaining a high index of suspicion, and establishing a diagnosis of Candida albicans ventriculoperitoneal shunt infection in the presence of one positive CSF culture in a symptomatic patient with an indwelling device, without discounting it as a contaminant. Treatment with systemic and intrathecal amphotericin-B or Fluconazole should be promptly started and can be switched to oral Fluconazole therapy for a total of 43 days. Follow-up CSF cytology and cultures should be performed, but elevated protein levels in the absence of other findings should not be solely used as an indicator of infection resolution and shunt function.

Further research is required in the form of a three-arm randomized or pragmatic clinical trial comparing the different decompression options - EVD, fontanelle taps, and serial lumbar punctures - in patients requiring temporary CSF drainage. External ventricular drainage makes inpatient care necessary and carries a high chance of overdrainage and nosocomial infections as compared to serial fontanelle taps. Ommaya reservoir is an alternative option that can be utilized for drainage of CSF as well as administration of intrathecal antibiotics. Nonetheless, fontanelle tap is emerging to be a good initial choice for neurosurgeons in a limited-resource setting as it requires a single tap to relieve the pressure, is more economical and manageable, can be carried out in an outpatient setup, provides a source for CSF challenge and has a lower risk of complications.

\section{Acknowledgements}

The authors would like to extend their gratitude and appreciation to Dr Fatima Mir, Assistant Professor of Pediatric Infectious Diseases, for her valuable input.

\section{References}

1. McClelland S, Hall WA (2007) Postoperative central nervous system infection: incidence and associated factors in 2111 neurosurgical procedures. Clin Infect Dis 45 55-59. [Crossref]

2. Schoenbaum SC, Gardner P, Shillito J (1975) Infections of cerebrospinal fluid shunts: epidemiology, clinical manifestations, and therapy. J Infect Dis 131: 543-552. [Crossref]

3. O'Brien D, Stevens NT, Lim CH, O'Brien DF, Smyth E, et al. (2011) Candida infection of the central nervous system following neurosurgery: a 12-year review. Acta Neurochir (Wien) 153: 1347-1350. [Crossref]

4. Baradkar VP, Mathur M, Sonavane A, Kumar S (2009) Candidal infections of ventriculoperitoneal shunts. J Pediatr Neurosci 4: 73-75. [Crossref]

5. Bayer AS, Edwards JE, Seidel JS, Guze LB (1976) Candida meningitis. Report of seven cases and review of the English literature. Medicine (Baltimore) 55: 477-486. [Crossref]

6. Chiou CC, Wong TT, Lin HH, Hwang B, Tang RB, et al. (1994) Fungal infection of ventriculoperitoneal shunts in children. Clin Infect Dis 19: 1049-1053. [Crossref]

7. Cruciani M, Di Perri G, Molesini M, Vento S, Concia E, et al. (1992) Use of fluconazole in the treatment of Candida albicans hydrocephalus shunt infection. Eur J Clin Microbiol Infect Dis 11: 957. [Crossref]

8. Forward KR, Fewer HD, Stiver HG (1983) Cerebrospinal fluid shunt infections. A review of 35 infections in 32 patients. J Neurosurg 59: 389-394. [Crossref]

9. Holt R (1969) The classification of staphylococci from colonized ventriculo-atrial shunts. J Clin Pathol 22: 475-482. [Crossref]

10. Jans J, Bruggemann RJ, Christmann V, Verweij PE, Warris A (2013) Favorable outcome of neonatal cerebrospinal fluid shunt-associated Candida meningitis with caspofungin. Antimicrob Agents Chemother 57: 2391-2393. [Crossref]

11. Murphy K, Bradley J, James HE (2000) The treatment of Candida albicans shun infections. Childs Nerv Syst 16: 4-7. [Crossref]

12. Rodgers BM, Vries JK, Talbert JL (1978) Laparoscopy in the diagnosis and treatment of malfunctioning ventriculo-peritoneal shunts in children. J Pediatr Surg 13: 247-253. [Crossref]

13. Shapiro S, Javed T, Mealey J Jr (1989) Candida albicans shunt infection. Pediatr Neurosci 15: 125-130. [Crossref]

14. Duhaime AC (2006) Evaluation and management of shunt infections in children with hydrocephalus. Clin Pediatr (Phila) 45: 705-713. [Crossref] 
Bhatti AU (2017) Candida albicans ventriculoperitoneal shunt infections: Immunocompetent preterm pediatric case series and an extensive review of literature

15. Kojic EM, Darouiche RO (2004) Candida infections of medical devices. Clin Microbiol Rev 17: 255-267. [Crossref]

16. Montero A, Romero J, Vargas JA, Regueiro CA, Sánchez-Aloz G, et al. (2000) Candida infection of cerebrospinal fluid shunt devices: report of two cases and review of the literature. Acta Neurochir (Wien) 142: 67-74. [Crossref]
17. Geers TA, Gordon SM (1999) Clinical significance of Candida species isolated from cerebrospinal fluid following neurosurgery. Clin Infect Dis 28: 1139-1147. [Crossref]

18. Tamber MS, Klimo P Jr, Mazzola CA, Flannery AM (2014) Pediatric Hydrocephalus Systematic Review and Evidence-Based Guidelines Task Force: Pediatric hydrocephalus: systematic literature review and evidence-based guidelines. Part 8: Management of cerebrospinal fluid shunt infection. J Neurosurg Pediatr 14: 60-71. [Crossref]

Copyright: (C2017 Bhatti AU. This is an open-access article distributed under the terms of the Creative Commons Attribution License, which permits unrestricted use, distribution, and reproduction in any medium, provided the original author and source are credited. 